\section{Near-field dielectric optics near the thermodynamic limit}

\author{
Daniel Feuermann, MEMBER SPIE ${ }^{a}$, Jeffrey M. Gordon ${ }^{a, b}$, \\ and Tuck Wah Ng, MEMBER SPIE \\ ${ }^{a}$ Ben-Gurion University of the Negev, Jacob Blaustein \\ Institutes for Desert Research, Department of Solar Energy \\ and Environmental Physics, Sede Boqer Campus \\ 84990, Israel \\ ${ }^{\mathrm{b}}$ Ben-Gurion University of the Negev, Department of \\ Mechanical Engineering, The Pearlstone Center \\ for Aeronautical Engineering Studies, Beersheva 84105, \\ Israel \\ ${ }^{\mathrm{C}}$ National University of Singapore, Faculty of Engineering, \\ Engineering Block EA-07-32, 9 Engineering Drive 1, \\ Singapore 117576 \\ E-mail: jeff@bgu.ac.il
}

Abstract. Practical aplanatic optics crafted from transparent dielectrics can approach the thermodynamic limit for radiative transfer in near-field systems. Designs are presented for the particularly challenging realm of high numerical aperture (NA) at the source and/or target. These light couplers can alleviate difficulties in aligning system components and can achieve the fundamental compactness limit for optical devices that satisfy Fermat's principle. Examples and performance estimates based on ray-trace simulations are presented. () 2006 Society of Photo-Optical Instrumentation Engineers. [DOI: $10.1117 / 1.2332812]$

Subject terms: optical design; optical systems; concentrators.

Paper 060239LR received Apr. 1, 2006; revised manuscript received May 24, 2006; accepted for publication May 30, 2006; published online Aug. 29, 2006.

\section{Introduction}

Radiative transfer near the thermodynamic limit ${ }^{1}$ is especially challenging for high-numerical-aperture (NA) systems - particularly with broadband light when chromatic aberration in refractive elements can be nonnegligible. A comprehensive investigation of the flux transfer properties of aplanatic ${ }^{2}$ optical systems for far-field designs $^{3}$ revealed classes of axisymmetric concentrators and illuminators that perform near the thermodynamic limit even at target NA values of unity (or a source NA of unity for illuminators), provided the angular radius of the farfield radiation is within around $20 \mathrm{mrad}$. The solutions were generalized for the more demanding near-field problem $^{4}$ (Fig. 1). These designs comprise two mirrors the contours of which eliminate spherical and comatic aberration, as first formulated in Ref. 2 but for which analyses were restricted to (1) image fidelity rather than radiative transfer and (2) air-filled, rather than all-dielectric, optics.

Dielectric-filled optical systems offer the pragmatic advantages of (1) mitigating alignment and orientation problems among the mirrors and target and (2) allowing molding and coating techniques that generate a monolithic optical system. Moreover, as a consequence of Snell's law, when the source is in air and the target is embedded in

0091-3286/2006/\$22.00 @ 2006 SPIE transparent dielectric, optical tolerance can be relaxed by a factor of $n$ (at fixed concentration, relative to air-filled systems), with $n \sim 1.5$ for feasible materials in visible and near-infrared applications. Optical tolerance comprises the convolution of contour and alignment errors with the finite size of the extended source. Alternatively, at fixed optical error, the dielectric allows increasing concentration by up to a factor of $n^{2}$, or smaller optics.

For example, a quasi-Lambertian disk (i.e., Lambertian emission up to a cutoff NA) of diameter $d$ has étendue $\left(\pi d^{2} / 4\right) \pi \mathrm{NA}^{2}=\left(\pi^{2} / 4\right) d^{2} n^{2} \sin ^{2}(\phi)$, where $\phi$ is the maximum half-angle in the medium. Performance near the thermodynamic limit refers to transferring close to all the source emission without the diameter of the target disc significantly exceeding its elemental minimum

$d_{\text {target }} \geq \frac{\mathrm{NA}_{\text {source }}}{\mathrm{NA}_{\text {target }}} d_{\text {source }}=\frac{n_{\text {source }} \sin \left(\phi_{\text {source }}\right)}{n_{\text {target }} \sin \left(\phi_{\text {target }}\right)} d_{\text {source }}$.

Equation (1) is sometimes referred to as the constrained thermodynamic limit, accommodating the general case where $\phi_{\text {target }}$ must be less than $90 \mathrm{deg}$.

Contoured refractive surfaces incur chromatic aberration, most notably in high-NA systems. Recently, a singlepiece, essentially achromatic far-field concentrator was proposed ${ }^{6}$ that entails filling an aplanatic design with dielectric and mirroring the exterior, such that first-surface mirror quality is attainable. Chromatic aberration is negligible because (1) the entry aperture is planar, with a sufficiently small angle subtended by the source at the optic (quantified in the following), and (2) light transfer is not contingent on total internal reflection. It was also shown that these aplanatic designs can reach the fundamental com-

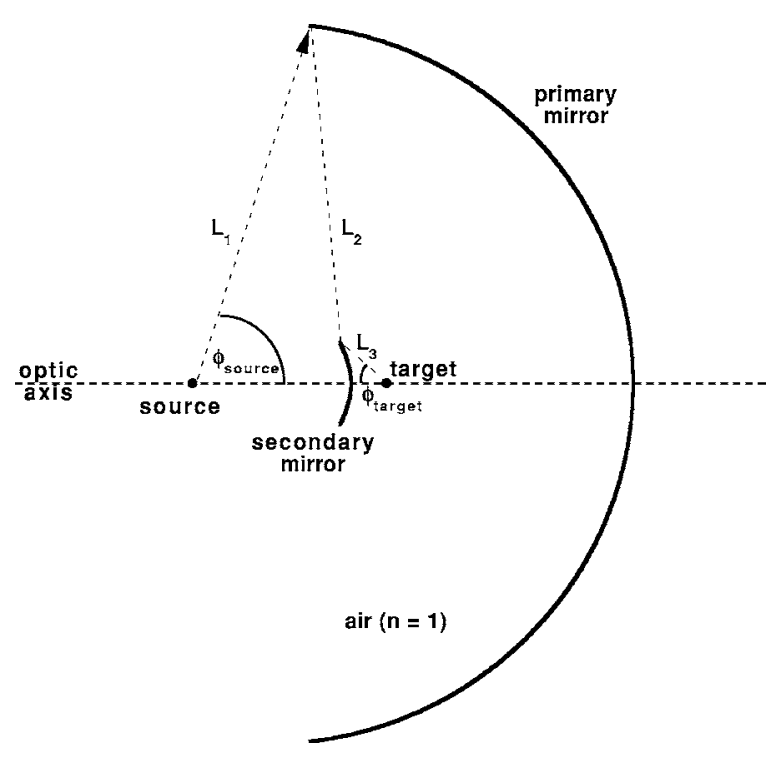

Fig. 1 Near-field (air-filled) axisymmetric aplanat, ${ }^{4}$ designed to image a point source of $N A_{\text {source }}=\sin \left(\phi_{\text {source }}\right)=0.95$ to a target focus of $N A_{\text {target }}=\sin \left(\phi_{\text {target }}\right)=0.66$. The closed-form solutions for the mirror contours ${ }^{4,5}$ follow from simultaneously satisfying: (1) Fermat's principle $L_{1}+L_{2}+L_{3}=$ const.; (2) Abbe's sine condition (constant magnification $m$ for all rays from a point source) $m$ $=\sin \left(\phi_{\text {source }}\right) / \sin \left(\phi_{\text {target }}\right)$; and (3) the law of specular reflection at the primary and secondary mirrors. 
pactness limit (an aspect ratio of 1/4) for any far-field optical system that satisfies Fermat's principle.

Here, we describe the analogous generalization to the near-field problem: a dielectric-filled optical system, essentially free of chromatic aberration, with performance approaching the thermodynamic limit. These devices can achieve a generalized compactness limit for near-field optical systems: a minimum aspect ratio that depends only on $\phi_{\text {source }}$. This study is motivated by applications of arc discharge lamps as alternatives to lasers for many fiber optic surgical procedures ${ }^{7}$ and is relevant to any system that imposes a sizable gap between source and concentrator. A discharge lamp emits over nearly the entire sphere. In applications such as photonic surgery, where only a single fiber is required, lamp emissions could be recycled back to the source, e.g., with a spherical mirror. ${ }^{7}$ Alternatively, multiple concentrator units of the type presented here could encompass the source. In addition, some LED-fiber and fiber-fiber couplings could benefit from near-field dielectric aplanats, which is why we include designs with the source embedded in the dielectric.

Near-field aplanatic solutions that constitute a dualmirror system were originally identified in Ref. 5 and analytic solutions were derived for the reflector contours. Their value as flux-transfer devices (concentrators and illuminators) were only explored recently, ${ }^{4}$ where it was demonstrated that performance can approach the thermodynamic limit even as the NA of the source and/or target approaches unity, provided the angular subtense from source to optical system is no larger than around $20 \mathrm{mrad}$. Only designs with shading not exceeding a few percent were analyzed to maintain practicality.

\section{Dielectric Optic with an Embedded Source}

One solution for a near-field all-dielectric device is to embed the entire system-source, aplanatic optic, and the space between them-in dielectric (Fig. 2). One retains the external mirror coatings. When it is feasible to embed the light source in the dielectric, $\mathrm{NA}_{\text {source }}$ can approach $n$. The choice of $\mathrm{NA}_{\text {target }}=0.66$ for the optical fiber is based on the highest NA for commercially available fibers which are

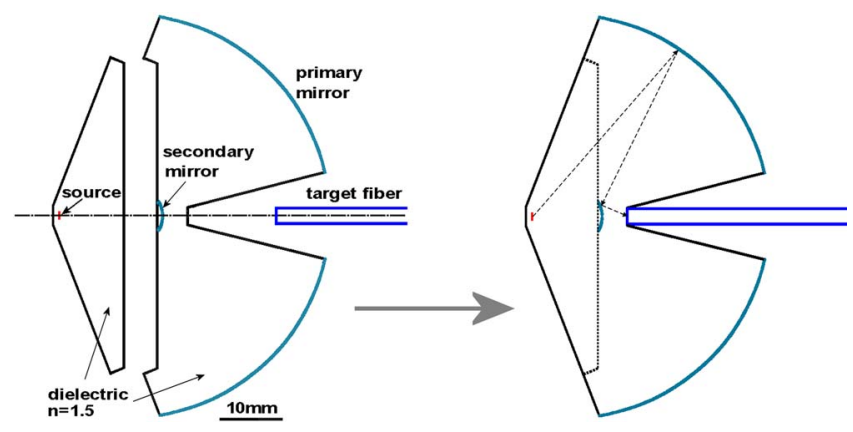

Fig. 2 System can be embedded in a dielectric of refractive index $n$ at no loss in efficiency, and remains essentially achromatic. Separate source and target units are prepared, joined, and optically coupled. The fiber here has a core refractive index $\approx n$, with $n=1.5$, $\mathrm{NA}_{\text {source }}=n \sin \left(\phi_{\text {source }}\right)=1.425, \quad N A_{\text {target }}=n \sin \left(\phi_{\text {target }}\right)=0.66, \quad$ and $\mathrm{NA}_{\text {target }}$ is chosen to match that of the optical fiber. Parameters are selected such that the rims of the primary and secondary mirrors are coplanar, and the device achieves the compactness limit described in the text. highly transmissive in the visible and near-infrared. ${ }^{8}$ To facilitate production and assembly of such units, the source and target sections could be manufactured separately and then combined (Fig. 2). Since a region much larger than the target area is shaded by the secondary, there is ample space for the insertion of an optical fiber target, including the cladding and buffer, or other channels for different applications.

\section{Design with the Source Removed}

A less obvious solution pertains to the pragmatic situation where either (1) the source is enclosed, as in discharge lamps, and the lamp should not touch or be embedded in a dielectric (so effectively $n_{\text {source }}=1$ ), or (2) the flexibility of operation or installation requires the source to be in air. Chromatic aberration can be essentially eliminated, and the optical integrity of the design maintained, by creating a spherical entry aperture (Fig. 3). The only refracting interface is the spherical entry, normal to rays from the source. The angular dispersion $\delta \theta$ at a point on the spherical entry where the source subtends half-angle $\theta$ is

$\delta \theta=-\tan (\theta) \delta n / n$,

which is negligible since $\theta \ll 1$. The optic is in effect achromatic.

In the illustrations, $n=1.5, d_{\text {source }}=1.0 \mathrm{~mm}$, and the halfangle subtended by the source at the rim of the primary is chosen as $\alpha=15 \mathrm{mrad}$ (hence the distance from the source to the rim of the primary is $33.3 \mathrm{~mm}$ ). Too large an angular subtense results in ray rejection from higher order aberrations. Smaller angular subtenses enlarge the device (concentrator linear dimensions scale as $1 / \alpha$ for $\alpha \ll 1$, so the mass of dielectric scales as $\left.1 / \alpha^{3}\right)$.

Flux transfer near the thermodynamic limit is realized (Fig. 4, for disk-to-disk transfer) in the sense that, except for geometric losses of a few percent, maximum radiative

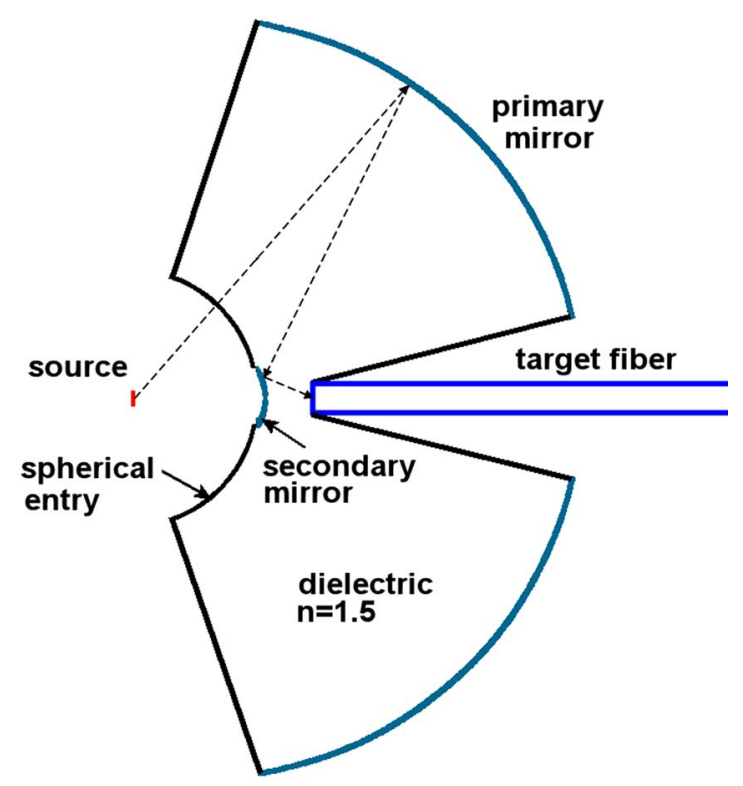

Fig. 3 Essentially achromatic near-field aplantic optics achieved with a spherical entry aperture. The source is in air; $\mathrm{NA}_{\text {source }}$ $=\sin \left(\phi_{\text {source }}\right)=0.95$; and $\mathrm{NA}_{\text {target }}=n \sin \left(\phi_{\text {target }}\right)=0.66$. 


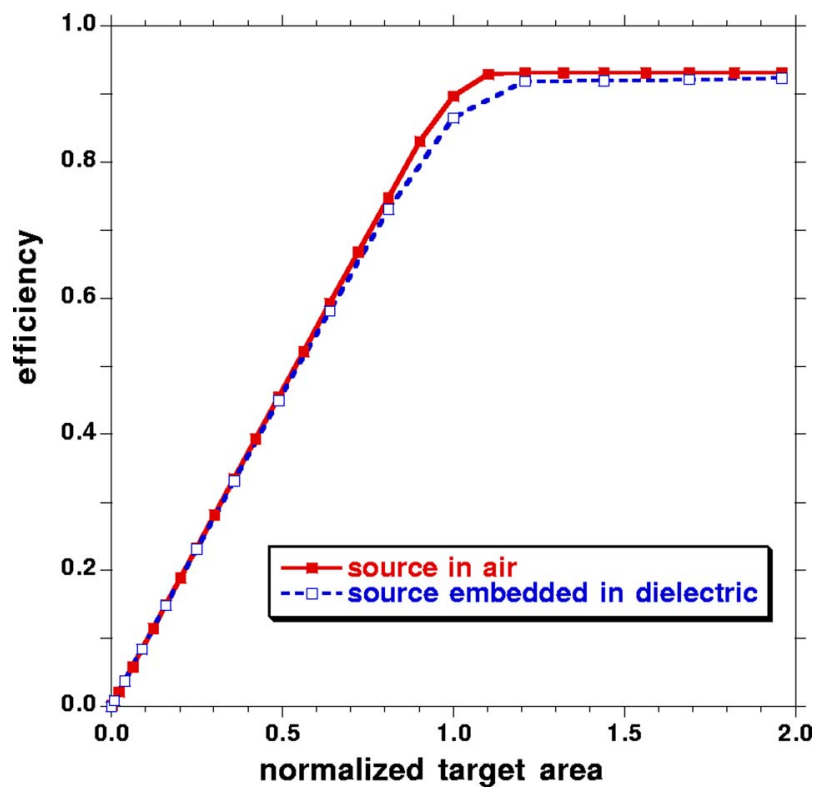

Fig. 4 Efficiency for the designs of Figs. 2 and 3 as a function of target area normalized to its minimum value [Eq. (1)]. Although the mirror contours are the same in both cases, the source size perceived inside the dielectric differs (as a consequence of Snell's law). Efficiency refers to the fraction of source emission reaching a target of given diameter (barring material-related losses), including ray rejection, shading, and blocking.

transfer is effected for the minimum absorber size commensurate with the étendue constraint of Eq. (1). For a discharge lamp source, the design remains nominally unaltered, but the target diameter must be adjusted in accordance with the étendue of the volumetric source. ${ }^{4}$ The prominent performance superiority of near-field (air-filled) aplanats relative to standard solutions such as ellipsoidal mirrors was quantified in Ref. 4. We find the same degree of performance advantage for these all-dielectric versions, in both the monolithic design of Fig. 2 and the source-in-air case of Fig. 3. Larger NA values are not considered because shading and ray rejection only increase ${ }^{4}$ with NA.

\section{Compactness Limit}

There is a compactness limit, in the near-field problem, for any optical device that satisfies Fermat's principle for all incident rays, derived here for a dual-mirror system in analogy to the corresponding far-field limit. ${ }^{6}$ Consider the type of devices portrayed in Figs. 1-3, with arbitrary relative placement of the primary and secondary along the optic axis, but satisfying the constraint of a selected input halfangle $\phi_{\text {source }}$. System aspect ratio (AR) is defined here as the quotient of (1) the distance between the source and the primary's apex, to (2) the diameter of the primary. Now trace a ray from the source to the focus via each of two points: (1) the rim of the primary and (2) along the optic axis. Stipulating constant optical path length to the focus, and requiring that $\mathrm{AR}$ be minimized, yields (1) that the primary and secondary be coplanar, as in Figs. 2 and 3 (coplanar meaning that the uppermost points of the primary and secondary mirrors lie in the same plane), and (2) $\mathrm{AR}_{\min }=(1 / 4)\left(1+\cot \left[\phi_{\text {source }} / 2\right]\right)$. Note $\mathrm{AR}_{\text {min }}$ rigorously corresponds to a vanishingly small secondary mirror, but the designs in Figs. 2 and 3 are examples that essentially achieve this limit.

\section{Concluding Remarks}

The challenge considered here is achieving flux transfer near the thermodynamic limit, in near-field systems, with a high-NA source and/or target requirement. The devices should be practical, as compact as possible, and effectively devoid of chromatic aberration. It turns out that dielectricfilled versions of a recently developed class of dualreflector aplanats satisfy all the criteria. Chromatic aberration is rendered negligible by either (1) embedding the entire system in a dielectric or (2) creating a spherical entry aperture to the dielectric that collects light from a source in air (essential in coupling light from a discharge lamp into a fiber).

The optical action does not rely on total internal reflection. Design and optimization are facilitated by the fact that the equations of all optical surfaces can be expressed in closed form. ${ }^{4,5}$ Also, the roles of source and target here can be interchangeable. Difficulties in the sensitive alignment of optical components are largely mitigated by the alldielectric designs.

\section{References}

1. R. Winston, J. C. Miñano, and P. Benítez, Nonimaging Optics, Elsevier Academic Press, Oxford (2005).

2. K. Schwarzschild, "Untersuchungen zur geometrischen Optik I-III," Abh. Akad. Wiss. Goettingen Math.-Phys. Kl. 4(1-3), 1 (1905-1906) (in German).

3. J. M. Gordon and D. Feuermann, "Optical performance at the thermodynamic limit with tailored imaging designs," Appl. Opt. 44, $2327-2331(2005)$

4. D. Nakar, D. Feuermann, and J. M. Gordon, "Aplanatic near-field optics for efficient light transfer," Opt. Eng. 45, 030502 (2006).

5. A. K. Head, "The two-mirror aplanat," Proc. Phys. Soc. London, Sect. B 70, 945-949 (1957).

6. R. Winston and J. M. Gordon, "Planar concentrators near the étendue limit," Opt. Lett. 30, 2617-2619 (2005).

7. D. Feuermann, J. M. Gordon, and T. W. Ng, "Photonic surgery with noncoherent light," Appl. Phys. Lett. 88, 114104 (2006).

8. D. Feuermann, J. M. Gordon, and M. Huleihil, "Light leakage in optical fibers: experimental results, modeling and the consequences for solar concentrators," Sol. Energy 72, 195-204 (2002). 